\title{
Search for $C P$ violation and rare decays in charm sector at Belle
}

\author{
Yun-Tsung Lai*i \\ KEK \\ E-mail: ytlai@post.kek.jp
}

Using more than $920 \mathrm{fb}^{-1}$ data collected with the Belle detector at the KEKB asymmetricenergy $e^{+} e^{-}$collider, we report the first measurement of the $T$-odd moments in the decay $D^{0} \rightarrow K_{S}^{0} \pi^{0} \pi^{+} \pi^{-}$. We search for $C P$-violation in decays $D^{0} \rightarrow K_{S}^{0} K_{S}^{0}$ and $D^{+} \rightarrow \pi^{+} \pi^{0}$. All the results are consistent with no $C P$ violation. We also report the result from the first search for $D^{0}$ decays to invisible final states. No significant signal yield is observed and an upper limit is set on the branching fraction at $90 \%$ confidence level.

The 39th International Conference on High Energy Physics (ICHEP2018)

4-11 July, 2018

Seoul, Korea

*Speaker.

†n behalf of the Belle Collaboration. 


\section{Search for $C P$ Violation and Measurement of the Branching Fraction in the $D^{0} \rightarrow K_{S}^{0} K_{S}^{0}$ Decay [1]}

$C P$ violation $(C P V)$ in charm meson decays is predicted to be $O\left(10^{-3}\right)$ in Standard Model (SM), and it has not been observed yet [2,3]. However, in the Single Cabibbo-Suppressed decays of $D$ mesons, possible interference with New Physics (NP) amplitude could lead to non-zero CPV [4], which could lead to physics beyond the SM.

The time-integrated $C P$ asymmetry $\left(A_{C P}\right)$ is defined as

$$
A_{C P}=\frac{\Gamma(D \rightarrow f)-\Gamma(\bar{D} \rightarrow \bar{f})}{\Gamma(D \rightarrow f)+\Gamma(\bar{D} \rightarrow \bar{f})}
$$

where $\Gamma$ is the partial decay width. $A_{C P}$ includes the terms due to direct $C P V$ and $D^{0}-\bar{D}^{0}$ mixing. The raw asymmetry $\left(A_{\text {raw }}\right)$ is measured with different flavors' cases:

$$
A_{\text {raw }}=\frac{N(D \rightarrow f)-N(\bar{D} \rightarrow \bar{f})}{N(D \rightarrow f)+N(\bar{D} \rightarrow \bar{f})}
$$

where $N$ is the fitted yield. $A_{\text {raw }}$ consists of $A_{C P}$ and other terms associated with detection efficiency of final-state particles. By measuring $A_{\text {raw }}$ of another decay $D \rightarrow f^{\prime}$ with well-measured $A_{C P}$, we can obtain $A_{C P}(D \rightarrow f)$ by the relation $\Delta A_{\text {raw }}\left(f, f^{\prime}\right)=\Delta A_{C P}\left(f, f^{\prime}\right) . D \rightarrow f^{\prime}$ can be utilized as the normalization mode for branching fraction measurement as well. We select $D^{0} \rightarrow K_{S}^{0} \pi^{0}$ as $D \rightarrow f^{\prime}$ in this study.

The $D^{* \pm}$ candidates are reconstructed with an addition $\pi_{\text {slow }}^{ \pm}$to identify $D^{0}$ 's flavor and to suppress combinatorial background. The signal yield is extracted by an unbinned extended maximum likelihood fit on $\Delta M \equiv M_{D^{* \pm}}-M_{D^{0}}$. The fit is done simultaneously for $D^{*+}$ and $D^{*-}$ cases. We obtain $\frac{\mathscr{B}\left(D^{0} \rightarrow K_{S}^{0} K_{S}^{0}\right)}{\mathscr{B}\left(D^{0} \rightarrow K_{S}^{0} \pi^{0}\right)}=(1.101 \pm 0.023) \%$ and $A_{C P}\left(D^{0} \rightarrow K_{S}^{0} K_{S}^{0}\right)=(-0.02 \pm 1.52) \%$, which is consistent with null asymmetry.

\section{Search for $C P$ Violation in the decay $D^{+} \rightarrow \pi^{+} \pi^{0}$ at Belle [5]}

$C P V$ in charm meson decays is expected to be small in the SM. However, in the world average of $\Delta A_{C P}\left(D^{0} \rightarrow K^{+} K^{-}, D^{0} \rightarrow \pi^{+} \pi^{-}\right)=(-0.656 \pm 0.154) \%$ [6], we found possible non-zero value in the difference, which could be a hint of NP. In addition, ref.[7] also suggests checking a sum rule related to three isospin related $D \rightarrow \pi \pi$ modes' asymmetry:

$$
R=\frac{A_{C P}\left(D^{0} \rightarrow \pi^{+} \pi^{-}\right)}{1+\frac{\tau_{D^{0}}}{\mathscr{B}_{+-}}\left(\frac{\mathscr{B}_{00}}{\tau_{D^{0}}}+\frac{2}{3} \frac{\mathscr{B}_{+0}}{\tau_{D^{+}}}\right)}+\frac{A_{C P}\left(D^{0} \rightarrow \pi^{0} \pi^{0}\right)}{1+\frac{\tau_{D^{0}}}{\mathscr{B}_{00}}\left(\frac{\mathscr{B}_{+-}}{\tau_{D^{0}}}+\frac{2}{3} \frac{\mathscr{B}_{+0}}{\tau_{D^{+}}}\right)}+\frac{A_{C P}\left(D^{+} \rightarrow \pi^{+} \pi^{0}\right)}{1+\frac{3}{2} \frac{\tau_{D^{+}}}{\mathscr{B}_{+0}}\left(\frac{\mathscr{B}_{00}}{\tau_{D^{0}}}+\frac{\mathscr{B}_{+-}}{\tau_{D^{0}}}\right)},
$$

where $\tau$ is the lifetime of $D$ mesons. If $R$ is consistent 0 while $A_{C P}\left(D^{+} \rightarrow \pi^{+} \pi^{0}\right)$ is not, it would be a hint of NP.

In this study, we select $D^{+} \rightarrow K_{S}^{0} \pi^{+}$as $D \rightarrow f^{\prime}$. Both the $D^{* \pm} \rightarrow D^{ \pm} \pi_{\text {slow }}^{0}$ candidates and the untagged $D^{ \pm}$candidates are included in the measurement to increase statistics. Signal yield is extracted by a fit on $M_{D^{ \pm}}$. We obtain $A_{C P}\left(D^{+} \rightarrow \pi^{+} \pi^{0}\right)=(+2.32 \pm 1.24 \pm 0.23) \%$ and $R=$ $(-2.2 \pm 2.7) \times 10^{-3}$. 


\section{First measurement of $T$-odd moments in $D^{0} \rightarrow K_{S}^{0} \pi^{0} \pi^{+} \pi^{-}$[8]}

The self-conjugated $D^{0} \rightarrow K_{S}^{0} \pi^{0} \pi^{+} \pi^{-}$can be used for a precise test of $C P V$, and the large statistics due to large branching fraction of 5.2\% [9] can enhance the precision of measurement with $O\left(10^{-3}\right)$. This decay is sensitive to $C P V$ via the $C P T$ theorem [10].

To measure $T$ violation $[11,12,13,14]$, two asymmetry parameters are defined by using scalar triple products $C_{T}=\mathbf{p}_{\mathbf{K}_{\mathbf{S}}^{0}} \cdot\left(\mathbf{p}_{\pi^{+}} \times \mathbf{p}_{\pi^{-}}\right)$and $\bar{C}_{T}=\mathbf{p}_{\mathbf{K}_{\mathbf{S}}^{\mathbf{0}}} \cdot\left(\mathbf{p}_{\pi^{-}} \times \mathbf{p}_{\pi^{+}}\right)$:

$$
A_{T}=\frac{\Gamma\left(C_{T}>0\right)-\Gamma\left(C_{T}<0\right)}{\Gamma\left(C_{T}>0\right)+\Gamma\left(C_{T}<0\right)}, \bar{A}_{T}=\frac{\Gamma\left(-\bar{C}_{T}>0\right)-\Gamma\left(-\bar{C}_{T}<0\right)}{\Gamma\left(-\bar{C}_{T}>0\right)+\Gamma\left(-\bar{C}_{T}<0\right)},
$$

for $D^{0}$ and $\bar{D}^{0}$. Non-zero value of $A_{T}$ or $\bar{A}_{T}$ could be due to the final state effect. To eliminate possible final state effect in $A_{T}$ and $\bar{A}_{T}$, we define

$$
a_{C P}^{T-\text { odd }}=\frac{1}{2}\left(A_{T}-\bar{A}_{T}\right) .
$$

Nonzero $a_{C P}^{T-\text { odd }}$ would indicate a clear $T$ violation.

$D^{* \pm} \rightarrow D^{0} \pi_{\text {slow }}^{ \pm}$is also reconstructed in this study. We perform a 2-dimensional fit on $\Delta M$ and $M_{D^{0}}$ of the four cases $\left(C_{T}>0, C_{T}<0,-\bar{C}_{T}>0\right.$, and $\left.-\bar{C}_{T}<0\right)$ simultaneously to obtain the yields of the four cases and the asymmetry parameters. We obtain $A_{T}=(11.60 \pm 0.19) \%$ and $a_{C P}^{T-\text { odd }}=\left(-0.28 \pm 1.38_{-0.76}^{+0.23}\right) \times 10^{-3}$. Further measurements in nine exclusive regions of the $K_{S}^{0} \pi^{0} \pi^{+} \pi^{-}$phase space with resonance $\left(K_{S}^{0} \omega, K_{S}^{0} \eta, K^{*-} \rho^{+}, K^{*+} \rho^{-}, K^{*-} \pi^{+} \pi^{0}, K^{*+} \pi^{-} \pi^{0}\right.$, $K^{*+} \pi^{+} \pi^{-}, K_{S}^{0} \rho^{+} \pi^{-}$, and the reminder) also show no evidence of $C P V$ in those bins.

\section{Search for $D^{0}$ decays to invisible final states at Belle [15]}

Branching fractions of $D$ decay to $v \bar{v}$ is helicity suppressed [16] in SM and is predicted as $1.1 \times 10^{-30}$. Under various types of Dark Matter models [16], branching fraction of $D$ decay to invisible final state can be enhanced to $O\left(10^{-15}\right)$.

To identify $D^{0}$ decay with invisible final state and to measure its absolute branching fraction, we utilize the charm tagger method $[17,18,19,20]$ to select an recoil $D^{0}$ sample by reconstructing the process $e^{+} e^{-} \rightarrow c \bar{c} \rightarrow D_{\text {tag }}^{(*)} X_{\text {frag }} \bar{D}_{\text {sig }}^{*-}$ with $\bar{D}_{\text {sig }}^{*-} \rightarrow \bar{D}_{\text {sig }}^{0} \pi_{\text {slow }}^{-}$, where $D_{\text {tag }}^{(*)}$ is a charm particle as a tag, $X_{\text {frag }}$ is the fragmentation system with a few light unflavored particles, $\pi_{\text {slow }}^{-}$is a charged pion from $\bar{D}_{\text {sig }}^{*-}$, and $\bar{D}_{\text {sig }}^{0}$ is the recoil $D^{0}$. By the fit on the $M_{D^{0}} \equiv M_{\text {miss }}\left(D_{\text {tag }}^{(*)} X_{\text {frag }} \pi_{\text {slow }}^{-}\right)$, we obtain $694505_{-1472}^{+1030}$ inclusive $D^{0}$ yield with Belle data.

Invisible $D^{0}$ decays are identified by requiring no remaining final-state particles (e.g. tracks, $\pi^{0}$, and $K_{L}^{0}$ ) associated with $\bar{D}_{\text {sig. }}^{0}$. In addition to $M_{D^{0}}$, we also use the residual energy in the Electromagnetic Calorimeter $\left(E_{\mathrm{ECL}}\right)$ to identify signals. A two-dimensional extended unbinned maximum likelihood fit on $M_{D^{0}}$ and $E_{\mathrm{ECL}}$ is performed, and we obtain a signal yield of $-10.2_{-20.8}^{+22.1}$. Since there is no significant yield observed, we set an upper limit of $8.8 \times 10^{-5}$ for $\mathscr{B}\left(D^{0} \rightarrow\right.$ invisible) at the $90 \%$ confidence level. 


\section{5. summary}

We report the $C P V$ measurement in $D^{0} \rightarrow K_{S}^{0} K_{S}^{0}$ and $D^{+} \rightarrow \pi^{+} \pi^{0}$ decays, first measurement of $T$-odd moments in $D^{0} \rightarrow K_{S}^{0} \pi^{0} \pi^{+} \pi^{-}$, and the first search for rare decay $D^{0}$ decays to invisible final states by using more than $920 \mathrm{fb}^{-1}$ data of Belle. All the $C P V$ results show null asymmetry, and we observe no significant yield for the $D^{0} \rightarrow$ invisible decay and we set an upper limit on the branching fraction at $90 \%$ confidence level for it. In Belle II, we expect 40 times of integrated luminosity. The $C P V$ and rare decays measurements of charm mesons can be further improved with higher precision.

\section{References}

[1] N. Dash et al. (Belle Collaboration), Phys. Rev. Lett. 119, 171801 (2017).

[2] Y. Grossman, A. L. Kagan, and Y. Nir, Phys. Rev. D 75, 036008 (2007).

[3] S. L. Glashow, J. Iliopoulos, and L. Maiani, Phys. Rev. D 2, 1285 (1970).

[4] G. Hiller, M. Jung, and S. Schacht, Phys. Rev. D 87, 014024 (2013).

[5] V. Badu et al. (Belle Collaboration), Phys. Rev. D 97, 011101 (2018).

[6] Y. Amhis et al. (Heavy Flavor Averaging Group), arXiv: 1207.1158.

[7] Y. Grossman, A. L. Kagan, and J. Zupan, Phys. Rev. D 85, 114036 (2012)

[8] K. Prasanth et al. (Belle Collaboration), Phys. Rev. D 95, 091101(R) (2018).

[9] M. Tanabashi et al. (Particle Data Group), Phys. Rev. D 98, 010001 (2018).

[10] G. Lüders, Det. Kong. Dansk. Vidensk. Selsk., Mat.-fys.Medd. 28, 005 (1954).

[11] J. M. Link et al. (FOCUS Collaboration), Phys. Lett. B 622, 239 (2005).

[12] P. del Amo Sanchez et al. (BABAR Collaboration), Phys. Rev. D 81, 111103 (2010).

[13] J. P. Lee et al. (BABAR Collaboration), Phys. Rev. D 84, 031103 (2011).

[14] R. Aaij et al. (LHCb Collaboration), J. High Energy Phys. 40 (2014) 005.

[15] Y.-T. Lai et al. (Belle Collaboration), Phys. Rev. D 95, 011102(R) (2017).

[16] A. Badin and A. A. Petrov, Phys. Rev. D 82, 034005 (2010).

[17] L. Widhalm et al. (Belle Collaboration), Phys. Rev. Lett. 97, 061804 (2006).

[18] L. Widhalm et al. (Belle Collaboration), Phys. Rev. Lett. 100, 241801 (2008).

[19] A. Zupanc et al. (Belle Collaboration), J. High Energy Phys. 09 (2013) 139.

[20] P. del Amo Sanchez et al. (BABAR Collaboration), Phys. Rev. D 82, 091103 (2010). 